\title{
FINDING THE ROOTS OF POLYNOMIAL EQUATIONS: AN ALGORITHM WITH LINEAR COMMAND
}

\author{
Bernard BEAUZAMY
}

\begin{abstract}
We show how an old principle, due to Walsh (1922), can be used in order to construct an algorithm which finds the roots of polynomials with complex coefficients. This algorithm uses a linear command. From the very first step, the zero is located inside a disk, so several zeros can be searched at the same time.
\end{abstract}

\section{Introduction}

Our algorithm will rely on an an old principle, due to Walsh ([3], 1922), which has not been employed as much as it should be. Indeed, "Walsh contraction principle", as we call it here, can be successfully employed in order to derive, more simply and with quantitative improvements, all known results about the zeros of a polynomial and those of its derivative, such as Lucas' Theorem, Grace's Theorem, Laguerre's Theorem and many others. These consequences will be presented elsewhere. Here, we concentrate on the search of the zeros.

The algorithm itself is, at this stage, purely theoretical: it works "in principle". It has been implemented, but has not been tested against other algorithms. A lot of work remains to be done, in a more numerical context, trying to find the situations in which a particular algorithm (including this one) does not work well, or, on the contrary, behaves satisfactorily. We thought it necessary to present first the theory.

In a first section, before we describe the algorithm, we give the statement and the proof of Walsh's principle [3].

1991 Mathematics Subject Classification: Primary 05A19, 11 C08.

Servicio de Publicaciones. Universidad Complutense. Madrid, 2000 


\section{Walsh's contraction principle}

Recall that a (closed) circular region is either a (closed) disk, or a (closed) half-plane, or the (closed) exterior of a disk. Any of the three can be transformed into one of the two others by a suitable map $z \rightarrow \frac{a z+b}{c z+d}$, $a d-b c \neq 0$.

These maps were called "linear" by Walsh ; they are now called "bilinear" or "homographic". They are indeed linear if one uses homogeneous coordinates: if $z=z_{1} / z_{2}$, then the new coordinates are $Z_{1}=$ $a z_{1}+b z_{2}, Z_{2}=c z_{1}+d z_{2}$. Such homogeneous coordinates are useful, in order to pass from a one variable polynomial $P(z)=\sum_{0}^{n} a_{j} z^{j}$ to the associated two-variable homogeneous polynomial $P\left(z_{1}, z_{2}\right)=\sum_{0}^{n} a_{j} z_{1}^{j} z_{2}^{n-j}$. The correspondence between both polynomials and between both systems of variables will be used several times in the sequel.

Theorem 1. (J.L. Walsh). Let $\varphi\left(z_{1}, \ldots, z_{n}\right)$ be a polynomial in $n$ variables, with the following properties :

$\varphi$ has degree 1 with respect to each variable,

$\varphi$ is symmetric with respect to the variables (that is, $\varphi$ in invariant under permutation of the variables).

Let $D$ be a circular region and assume that in $D$ there are points $\left(z_{1}, \ldots, z_{n}\right)$ such that

$$
\varphi\left(z_{1}, \ldots, z_{n}\right)=0 .
$$

Then there is in $D$ a point $z$ such that

$$
\varphi(z, \ldots, z)=0 .
$$

Proof. The proof we give follows the original one, with only slight improvements, but a more modern terminology. We may restrict ourselves to the case when $D$ is a disk, for any circular region can be transformed into a disk by a suitable homographic map, which will not modify the properties of $\varphi$.

The key to the proof is the following proposition :

Proposition 2. Let $a, b, c$ be three complex numbers. We consider the complex numbers $z=\left(z_{1}, z_{2}\right)$ (in homogeneous coordinates) solutions of 
the equation

$$
a z_{1}^{2}+2 b z_{1} z_{2}+c z_{2}^{2}=0 .
$$

Let $z^{\prime}=\left(z_{1}^{\prime}, z_{2}^{\prime}\right), z^{\prime \prime}=\left(z_{1}^{\prime \prime}, z_{2}^{\prime \prime}\right)$ be the two solutions of (1). Let also $u=\left(u_{1}, u_{2}\right), v=\left(v_{1}, v_{2}\right)$ be two complex numbers, satisfying

$$
a u_{1} v_{1}+b\left(u_{1} v_{2}+u_{2} v_{1}\right)+c u_{2} v_{2}=0 \text {. }
$$

Then the four points $z^{\prime}, u, z^{\prime \prime}, v$ are on the same circle (maybe degenerate) and in this order.

Proof of Proposition 2. General case: $b^{2}-a c \neq 0$. We define a linear transformation of homogeneous coordinates, by

$$
Z_{1}=\alpha z_{1}+\beta z_{2}, \quad Z_{2}=\gamma z_{1}+\delta z_{2} .
$$

We will see that, by a suitable choice of $\alpha, \beta, \gamma, \delta, \alpha \delta-\beta \gamma \neq 0$, (1) becomes $Z_{1}^{2}=Z_{2}^{2}$ and (2) becomes $U_{1} V_{1}=U_{2} V_{2}$.

Indeed, $Z_{1}^{2}=Z_{2}^{2}$ is equivalent to

$$
\left(\alpha z_{1}+\beta z_{2}\right)^{2}=\left(\gamma z_{1}+\delta z_{2}\right)^{2},
$$

that is

$$
\left(\alpha^{2}-\gamma^{2}\right) z_{1}^{2}+2(\alpha \beta-\gamma \delta) z_{1} z_{2}+\left(\beta^{2}-\delta^{2}\right) z_{2}^{2}=0,
$$

and $U_{1} V_{1}=U_{2} V_{2}$ is equivalent to

$$
\left(\alpha u_{1}+\beta u_{2}\right)\left(\alpha v_{1}+\beta v_{2}\right)=\left(\gamma u_{1}+\delta u_{2}\right)\left(\gamma v_{1}+\delta v_{2}\right),
$$

or

$$
\left(\alpha^{2}-\gamma^{2}\right) u_{1} v_{1}+(\alpha \beta-\gamma \delta)\left(u_{1} v_{2}+u_{2} v_{1}\right)+\left(\beta^{2}-\delta^{2}\right) u_{2} v_{2}=0 .
$$

So we take $\alpha, \beta, \gamma, \delta$ so that

$$
\alpha^{2}-\gamma^{2}=a, \quad \alpha \beta-\gamma \delta=b, \quad \beta^{2}-\delta^{2}=c,
$$

and such a choice of $\alpha, \beta, \gamma, \delta$ with $\alpha \delta-\beta \gamma \neq 0$ always exists, no matter what are the values of $a, b, c$, when $b^{2}-a c \neq 0$. 
We now consider the complex numbers $z^{\prime}, z^{\prime \prime}$, solutions of

$$
z_{1}^{2}=z_{2}^{2},
$$

and two complex numbers $u, v$ satisfying

$$
u_{1} v_{1}=u_{2} v_{2},
$$

and we will show that $z^{\prime}, u, z^{\prime \prime}, v$ are on the same circle, in this order.

Let $z_{1}=\xi_{1}+i \eta_{1}, z_{2}=\xi_{2}+i \eta_{2}$, where $\xi_{1}, \eta_{1}, \xi_{2}, \eta_{2} \in \mathbb{R}$.

A circle passing through $z=1$ and $z=-1$ is of the form

$$
|z-i \lambda|^{2}=1+\lambda^{2} \quad(\lambda \in \mathbb{R}),
$$

or, in homogeneous coordinates :

$$
\left|z_{1}-i \lambda z_{2}\right|^{2}=\left(1+\lambda^{2}\right)\left|z_{2}\right|^{2} .
$$

In homogeneous real coordinates, this gives :

$$
\left(\xi_{1}+\lambda \eta_{2}\right)^{2}+\left(\eta_{1}-\lambda \xi_{2}\right)^{2}=\xi_{2}^{2}+\eta_{2}^{2}+\lambda^{2} \xi_{2}^{2}+\lambda^{2} \eta_{2}^{2},
$$

or

$$
\xi_{1}^{2}+\eta_{1}^{2}+2 \lambda\left(\xi_{1} \eta_{2}-\eta_{1} \xi_{2}\right)=\xi_{2}^{2}+\eta_{2}^{2} .
$$

This equation in invariant if we exchange the indexes 1 and 2. Therefore, if it is satisfied by $u$, it is also satisfied by $v$, if $u v=1$. The order of points is clear, since if $u v=1, \arg u=-\arg v$.

-Now, let's look at the special cases where $b^{2}=a c$.

First, if $b \neq 0$ (so $a \neq 0$ and $c \neq 0$ ), (1) becomes $a z_{1}+b z_{2}=0$, and (2) becomes $\left(a u_{1}+b u_{2}\right)\left(a v_{1}+b v_{2}\right)=0$. So $z^{\prime}=z^{\prime \prime}$ and either $u$ or $v$ (or both) coïncide with $z^{\prime}$ and $z^{\prime \prime}$.

If $b=0$, and $a=0$ but $c \neq 0$, (1) becomes $z_{2}=0$ and (2) becomes $u_{2} v_{2}=0$, so $z^{\prime}=z^{\prime \prime}=\infty$ and either $u$ or $v$ is $\infty$.

If $b=0, a \neq 0, c=0,(1)$ is $z=0,(2)$ is $u_{1} v_{1}=0$, so $z^{\prime}=z^{\prime \prime}=0$ and $u$ or $v$ is 0 .

In all these cases, the line passing through $u=z^{\prime}=z^{\prime \prime}$ and $v$ (or by $v=z^{\prime}=z^{\prime \prime}$ and $u$ ) meets our request. 
Lemma 3. Let $z^{\prime}, z^{\prime \prime}, u, v$ be as in the assumptions of Proposition 2. Any circular region containing both $u$ and $v$ must contain either $z^{\prime}$ or $z^{\prime \prime}$.

Proof. This is obvious in the special cases. In the general case, by the previous transformation, we reduce ourselves to the problem: Any closed disk containing both $z$ and $1 / z$ must contain either 1 or -1 . But since $-1, z, 1,1 / z$ are on the same circle, in this order, this is obvious.

For $u, v, u \neq v$, satisfying (2), we have seen that there is a welldefined circle, passing through the four points $u, v$ and the two solutions of (1). If $u$ moves on this circle in one direction, $v$ moves in the other (still keeping (2) satisfied) and both points meet either at $z^{\prime}$ or at $z^{\prime \prime}$.

This circle, along which both $u, v$ can move, will be called their "circle of indifference" (it may be degenerate).

We now prove Walsh's theorem. Let $D$ be a circular region (it is enough to consider a closed disk) and $z_{1}, \ldots, z_{n} \in D$, with $\varphi\left(z_{1}, \ldots, z_{n}\right)=$ 0 . Let $D_{0} \subset D$ be the smallest closed disk for which there exist points $z_{1}^{\prime} \ldots, z_{n}^{\prime} \in D_{0}$, with $\varphi\left(z_{1}^{\prime}, \ldots, z_{n}^{\prime}\right)=0$. We are going to show that $D_{0}$ is reduced to a single point.

We assume that $D_{0}$ has non-empty interior, and we will reach a contradiction.

Let $C_{0}$ be the circle, boundary of $D_{0}$. We consider several cases :

1) If one of the $z_{i}^{\prime}$, say $z_{1}^{\prime}$, is not on $C_{0}$, the indifference circle of the pair $\left(z_{1}^{\prime}, z_{2}^{\prime}\right)$ cannot be $C_{0}$. We consider all pairs $\left(z_{1}^{\prime}, z_{i}^{\prime}\right)$ for which the second point $z_{i}^{\prime}$ is on $C_{0}$ (this must happen for at least one point, otherwise $D_{0}$ would not be minimal). Say for instance that $z_{2}^{\prime} \in C_{0}$. Then we can move both $z_{1}^{\prime}$ and $z_{2}^{\prime}$ on their indifference circle, so that $z_{2}^{\prime}$ is now in $D_{0}^{0}$ (the interior of $D_{0}$ ) and $z_{1}^{\prime}$ remains inside this interior (this is possible because the indifference circle of the pair is precisely not $C_{0}$ ). We proceed the same way with the new $z_{1}^{\prime}$ and $z_{3}^{\prime}$ (if $z_{3}^{\prime} \in C_{0}$ ) ; we omit the pairs $\left(z_{1}^{\prime}, z_{i}^{\prime}\right)$ for which $z_{i}^{\prime} \in D_{0}^{0}$. When we finish, all points are in 
the interior $D_{0}^{0}$, and $D_{0}$ was not minimal (fig.1).

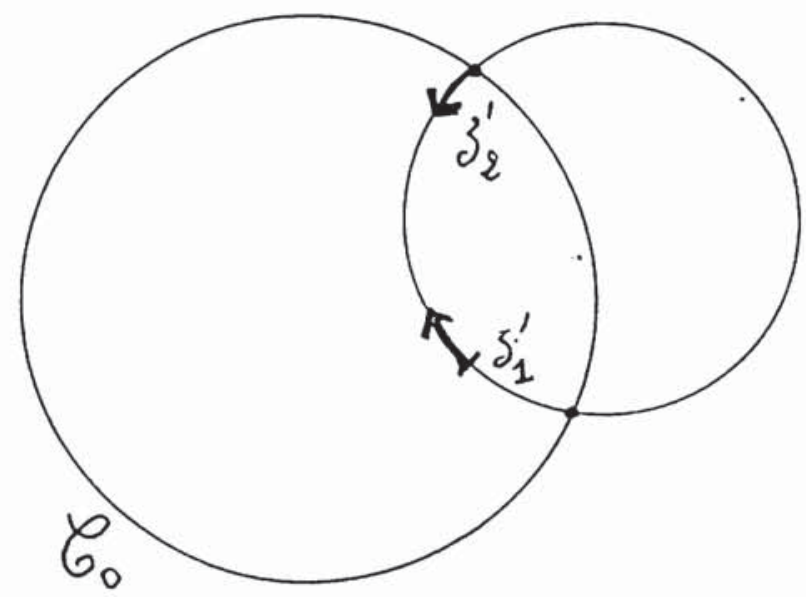

Figure 1

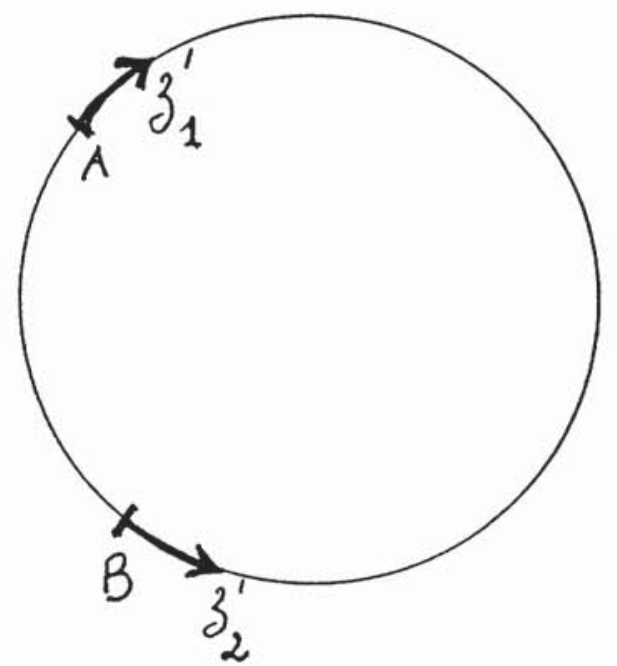

Figure 2

2) We assume now that all points $z_{i}^{\prime}$, are on $C_{0}$. If for some pair, say $\left(z_{1}^{\prime}, z_{2}^{\prime}\right)$, the indifference circle is not $C_{0}$, then both points can be moved on this indifference circle, so as to get inside $D_{0}^{0}$, and we are back to the situation of 1). So we can restrict ourselves, now, to the case where all 
points are on $C_{0}$, and moreover all pairs have $C_{0}$ as indifference circle (at this stage of the construction, or at later stages).

Let now $L_{0}$ be the smallest closed arc (in length) on $C_{0}$, containing all points $z_{1}^{\prime}, \ldots, z_{n}^{\prime}$ ( $L_{0}$ is simply the complement of the largest open arc between two consecutive points). Let $A, B \in C_{0}$ be the endpoints of $L_{0}$. Of course, some of the points $z_{i}^{\prime}$ are in $A$ (say $z_{1}^{\prime}, z_{2}^{\prime}, \ldots$ ), some are in $B$ (say $z_{n}^{\prime}, z_{n-1}^{\prime}, \ldots$ ). We take these points two by two : $z_{1}^{\prime}$ with $z_{n}^{\prime}$ to start with, and move them inside $L_{0}$. This is possible, since $C_{0}$ is precisely the indifference circle of $\left(z_{1}^{\prime}, z_{n}^{\prime}\right)$. When we are done with this pair, we pass to the next one, $\left(z_{2}^{\prime}, z_{n-1}^{\prime}\right)$, and so on. When we finish, at least one of the points $A$ or $B$ will be free from all $z_{i}^{\prime}$, and we see that $L_{0}$ was not minimal (see fig. 2). This contradiction finishes the proof.

As we just saw, the entire proof relies on a manipulation of pairs, playing with points two by two, and this is why the induction procedure is rather complicated.

So a natural question is : can't we take the $n$ points at once, and "shrink" them, still staying inside the given disk $D$ ? The answer is that such a manipulation is impossible in general. There are cases where manipulating three points at the same time obliges us to leave $D$. Indeed consider

$$
\varphi\left(z_{1}, z_{2}, z_{3}\right)=8 z_{1} z_{2} z_{3}-3\left(z_{1} z_{2}+z_{2} z_{3}+z_{3} z_{1}\right)+2\left(z_{1}+z_{2}+z_{3}\right)-2 .
$$

The equation $\varphi=0$ is satisfied for $z_{1}=z_{2}=0, z_{3}=1$.

Take now $z_{1}=z_{2}=x, z_{3}=y$, and let $D$ be the disk with diameter $[0,1]$. Then no manipulation of $x$ or of $y$, satisfying $\varphi(x, x, y)=0$, can leave them both in $D$. Indeed,

$$
\left|y-\frac{1}{2}\right|=\frac{1}{2}|x+1|^{2}\left|1-\frac{5 x^{3}}{(x+1)^{2}}+\frac{x^{3} \varepsilon(x)}{(x+1)^{2}}\right|, \quad \varepsilon \rightarrow 0, x \rightarrow 0,
$$

so to decrease $\left|y-\frac{1}{2}\right|$ means to decrease $|x+1|$. But one cannot decrease at the same time $\left|x-\frac{1}{2}\right|$ and $|x+1|$, starting at $x=0$.

The construction of this example will become clear when we study the algorithm itself. 


\section{Searching for the solutions of polynomial equa- tions: The theory}

We start with an $n$-degree polynomial in one variable, with complex coefficients

$$
P(z)=z^{n}+a_{n-1} z^{n-1}+\cdots+a_{k} z^{k}+\cdots+a_{0},
$$

and we want to solve the equation $P(z)=0$.

First, from $P(z)$, we construct a polynomial $P\left(x_{1}, \ldots, x_{n}\right)$ in $n$ variables, symmetric, and of degree 1 with respect to each variable. Moreover, $P(z, \ldots, z)=P(z)$. This polynomial is defined by

$$
\begin{aligned}
& P\left(x_{1}, \ldots, x_{n}\right)= \\
& x_{1} \cdots x_{n}+\frac{a_{n-1}}{n} \sum_{i_{1}<\ldots<i_{n-1}} x_{i_{1}} \cdots x_{i_{n-1}} \\
& +\cdots+\frac{a_{k}}{\left(\begin{array}{c}
n \\
k
\end{array}\right)} \sum_{i_{1}<\ldots<i_{k}} x_{i_{1}} \cdots x_{i_{k}}+\cdots+a_{0}
\end{aligned}
$$

and Walsh's principle tells us that in any disk containing a $n$-tuple $\left(x_{1}, \ldots, x_{n}\right)$ satisfying

$$
P\left(x_{1}, \ldots, x_{n}\right)=0
$$

there is a $z$ satisfying (1), that is a solution of the polynomial equation.

We will take the first $n-1$ variables at the same place, say $z_{1}=$ $\cdots=z_{n-1}=x$, and the last one, say $z_{n}=y$, will be deduced from (2). This way, the dependence in $y$ will be linear, and we will obtain what we call a "linear command". With this choice of points, (2) becomes :

$$
\begin{aligned}
& \left.x^{n-1} y+\frac{a_{n-1}}{n}\left(\begin{array}{c}
n-1 \\
n-1
\end{array}\right) x^{n-1}+\left(\begin{array}{c}
n-1 \\
n-2
\end{array}\right) x^{n-2} y\right)+\cdots+ \\
& \frac{a_{k}}{\left(\begin{array}{c}
n \\
k
\end{array}\right)}\left(\left(\begin{array}{c}
n-1 \\
k
\end{array}\right) x^{k}+\left(\begin{array}{c}
n-1 \\
k-1
\end{array}\right) x^{k-1} y\right)+\cdots+a_{0}=0,
\end{aligned}
$$

or

$$
\begin{aligned}
& x^{n-1} y+\frac{a_{n-1}}{n}\left(x^{n-1}+(n-1) x^{n-2} y\right) \\
& +\cdots+\frac{a_{k}}{n}\left((n-k) x^{k}+k x^{k-1} y\right)+\cdots+a_{0}=0
\end{aligned}
$$


that is

$$
\begin{gathered}
\left(\sum_{k=0}^{n} k a_{k} x^{k-1}\right) y+\sum_{0}^{n}(n-k) a_{k} x^{k}=0, \\
\left(\sum_{k=0}^{n} k a_{k} x^{k-1}\right) y=-n \sum_{0}^{n} a_{k} x^{k}+\sum_{0}^{n} k a_{k} x^{k},
\end{gathered}
$$

which can be written

$$
n P(x)+(y-x) P^{\prime}(x)=0
$$

and means that

$$
P_{1}(y, x)=0,
$$

where $P_{1}(y, x)$ is the polar derivative of $P$ at the point $y$ (recall that this polar derivative is defined by

$$
P_{1}(y, x)=n P(x)+(y-x) P^{\prime}(x),
$$

see Marden [2, p. 49] and Beauzamy-Dégot [1]).

If $P^{\prime}(x) \neq 0$, we obtain the equation

$$
y=x-n \frac{P(x)}{P^{\prime}(x)} .
$$

Remark. Once again, equations (4), (5), (6) are perhaps better understood if we use the homogeneous two-variable polynomial associated with $P$. Indeed, let

$$
P\left(x_{1}, x_{2}\right)=x_{1}^{n}+a_{n-1} x_{1}^{n-1} x_{2}+\cdots+a_{k} x_{1}^{k} x_{2}^{n-k}+\cdots+a_{0} x_{2}^{n} .
$$

Then, let $x=x_{1} / x_{2}, y=y_{1} / y_{2}$ be homogeneous coordinates. We obtain

$$
y_{1} \frac{\partial P}{\partial x_{1}}\left(x_{1}, x_{2}\right)+y_{2} \frac{\partial P}{\partial x_{2}}\left(x_{1}, x_{2}\right)=0,
$$

which is another expression of the polar derivative (see [1]).

We obtain easily a preliminary information on the location of the zeros of $P$ :

Proposition 4. Let $x$ be any point such that $P^{\prime}(x) \neq 0$, and let $y=$ $x-n P(x) / P^{\prime}(x)$. Then the closed disk of diameter $[x, y]$ contains at least one zero of $P$. 
Proof. This is a just a consequence of Walsh's principle.

Of course, if the initial point $x$ satisfies $P^{\prime}(x)=0$, we change it for another one (recall that the zeros of $P^{\prime}$ are called "critical points" of $P$ by Walsh [3]).

We now aim at decreasing the distance $|x-y|$, in order to, eventually, satisfy $x=y$. For this, we replace $x$ by $x+d x, y$ by $y+d y$. Equation (6) gives, by differentiation :

$$
d y=\left(1-n \frac{P^{\prime 2}-P P^{\prime \prime}}{P^{\prime 2}}(x)\right) d x,
$$

so we see that the increment $d y$, applied to $y$, depends linearly on the command $d x$ (thus justifying our title).

We may meet two situations : either $P^{\prime 2}(x)-P(x) P^{\prime \prime}(x) \neq 0$ (then $x$ will be called a regular point), or $P^{\prime 2}(x)-P(x) P^{\prime \prime}(x)=0$ (then $x$ is a singular point, or as we will prefer later, a point of multiple choices). Note that $P^{\prime 2}-P P^{\prime \prime}$ is a polynomial of degree $2 n-2$, so there are $2 n-2$ singular points in the plane.

a) If we are at a regular point, $P^{\prime 2}(x)-P(x) P^{\prime \prime}(x) \neq 0$, and

$$
(y+d y)-(x+d x)=y-x-n \frac{P^{\prime 2}(x)-P(x) P^{\prime \prime}(x)}{P^{\prime 2}(x)} d x .
$$

We write

$$
\begin{gathered}
F(x)=\frac{P(x)}{P^{\prime}(x)}, \\
F^{\prime}(x)=\frac{P^{\prime 2}(x)-P(x) P^{\prime \prime}(x)}{P^{\prime 2}(x)},
\end{gathered}
$$

and we let $\theta=\operatorname{Arg} F^{\prime}(x), \theta_{1}=\operatorname{Arg}(y-x)$. We will choose the increment $d x$ in such a way that

$$
\left|y-x-n F^{\prime}(x) d x\right|<|y-x| .
$$

This is the case if $y-x$ and $n F^{\prime}(x) d x$ are on the same line, in the same direction, that is

$$
\theta_{1}=\theta+\operatorname{Arg}(d x)
$$


or

$$
\operatorname{Arg}(d x)=\theta_{1}-\theta .
$$

Then (9) holds as soon as $|d x|$ is small enough ; a precise value can be derived from Taylor formula, as follows.

With $x^{\prime}=x+d x$, and the argument $\psi$ of $d x$ fixed as explained earlier, we have :

$$
F\left(x^{\prime}\right)=-\frac{1}{n}(y-x)+\left(x^{\prime}-x\right) F^{\prime}(x)+\frac{\left(x^{\prime}-x\right)^{2}}{2} F^{\prime \prime}(c)
$$

for some $c$ on the segment $\left[x, x^{\prime}\right]$.

Therefore, if $d x=\delta e^{i \psi}$,

$$
\left|F\left(x^{\prime}\right)\right| \leq\left|-\frac{1}{n}(y-x)+\left(x^{\prime}-x\right) F^{\prime}(x)\right|+\frac{\delta^{2}}{2}\left|F^{\prime \prime}(c)\right|
$$

and with $M_{\delta}=\sup \left\{\left|F^{\prime \prime}(x+\eta)\right| ;|\eta| \leq \delta\right\}$,

$$
\left|F\left(x^{\prime}\right)\right| \leq \frac{|y-x|}{n}-\delta\left|F^{\prime}(x)\right|+\frac{\delta^{2}}{2} M_{\delta}
$$

and this is smaller than $|y-x| / n$ as soon as

$$
\delta M_{\delta} \leq 2\left|F^{\prime}(x)\right|
$$

A precise bound depends on $M_{\delta}$. In practice, we will take

$$
|d x|=\frac{|y-x|}{n\left|F^{\prime}(x)\right|} .
$$

b) If we are at a singular point, the first derivative of $F$ is zero. This means that, in the first order of approximation, $|y-x|$ does not change, no matter in which direction $x$ moves.

Let's first consider an example : $P(z)=z^{2}+1, P^{\prime}(z)=2 z, F(x)=$ $\frac{x^{2}+1}{2 x}$, and $F^{\prime}=\frac{x^{2}-1}{2 x^{2}}$. 
So the point $x=1$, giving $y=-1$ (since $x y=-1$ ), is a singular point, or more exactly, a point of multiple choices :

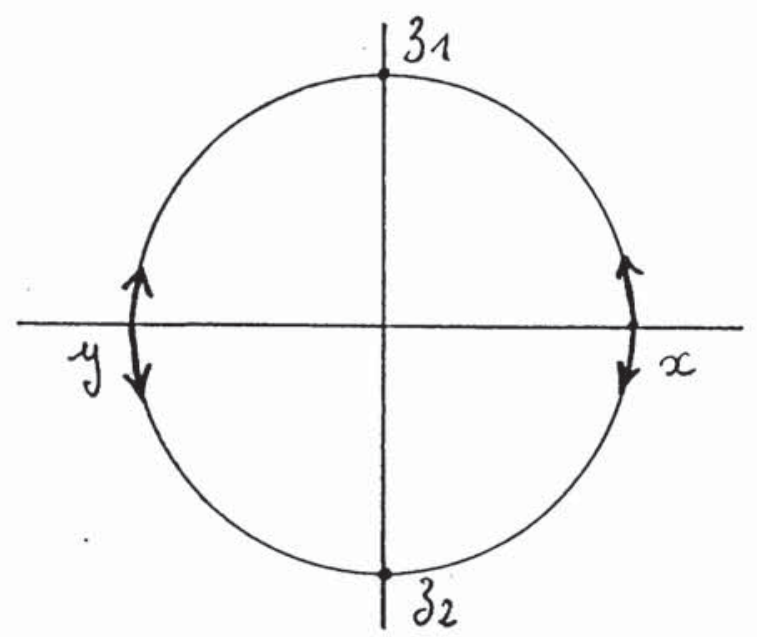

Figure 3

You may choose to move upwards (in order to catch $z_{1}$ ) and $y$ will move accordingly, or downwards, and $y$ will do the same: If $x=e^{i \alpha}$, $y=e^{-i \alpha}$.

It should be understood on this example that singular points are not bad points. There are places at which one has to make a choice; they are, in some sense, boundaries between basins of attraction associated with the zeros. The easiest way to get out of the ambiguity would be to make a small move at random, in any direction, but there are better ways, as we will now see.

Lemma 5. A point $x$ such that

$$
F^{\prime}(x)=F^{\prime \prime}(x)=\cdots=F^{(k)}(x)=0
$$

satisfies

$$
\frac{P^{\prime}(x)}{P(x)}=\frac{P^{\prime \prime}(x)}{P^{\prime}(x)}=\cdots=\frac{P^{(k+1)}(x)}{P^{(k)}(x)} .
$$


Proof of Lemma 5. If, at the point $x, F$ is "flat at the order $k$ ", that is satisfies $F^{\prime}=\cdots=F^{(k)}=0$, so is $1 / F=P^{\prime} / P$ For $k=1$, we get :

$$
\left(P^{\prime} / P\right)^{\prime}=\frac{P^{\prime \prime}}{P}-\left(\frac{P^{\prime}}{P}\right)^{2}=\frac{P^{\prime}}{P}\left(\frac{P^{\prime \prime}}{P^{\prime}}-\frac{P^{\prime}}{P}\right),
$$

and since we are at a point where $P^{\prime}(x) \neq 0,(12)$ follows. Now, in (13), all terms are sums or products of terms of the form $\frac{f^{\prime}}{f}$, and this will be preserved under derivation. When computing $F^{(k)}$, we get only one term of the form $\frac{P^{(k+1)}}{P^{(k)}}-\frac{P^{(k)}}{P^{(k-1)}}$. If we assume (12) up to the order $k-1$, all other terms disappear, and so this one must be zero. This proves the lemma.

So, since we are at a point where $F^{\prime}(x) \neq 0$, we must have $k<n$, and one of the derivatives $F^{\prime}(x), \ldots, F^{n-1}(x)$ must be $\neq 0$.

Say for instance that $F^{\prime \prime}(x) \neq 0$. Then, from

$$
y=x-n F(x),
$$

we deduce that, for the new positions $x+d x, y+d y$ :

$$
(y+d y)-(x+d x) \sim y-x-n \frac{(d x)^{2}}{2} F^{\prime \prime}(x)
$$

and we must take the argument of $d x$ in such a way that

$$
\operatorname{Arg}(y-x)=\operatorname{Arg}(d x)^{2}+\operatorname{Arg} F^{\prime \prime}(x),
$$

that is

$$
\operatorname{Arg}(d x)=\frac{1}{2}\left(\theta_{1}-\operatorname{Arg} F^{\prime \prime}(x)\right)
$$

and $|d x|$ small enough so that (14) holds. In practice :

$$
|d x|=\left(\frac{2|y-n|}{n\left|F^{\prime \prime}(x)\right|}\right)^{1 / 2} .
$$

A more geometrical method can be taken. If $F^{\prime}(x)=0$ but $F^{\prime \prime}(x) \neq$ $0, F$ is an analytic function which, locally, multiplies the angles by a factor 2 (if $F^{\prime} \neq 0, F$ is conformal and preserves the angles). 
Take any straight line passing through $x$ (say for instance the parallel to the $x$ axis) and the four directions $\theta=0, \theta=\pi / 4, \theta=\pi / 2, \theta=3 \pi / 4$. Their image is a cross with angles $\pi / 2$.
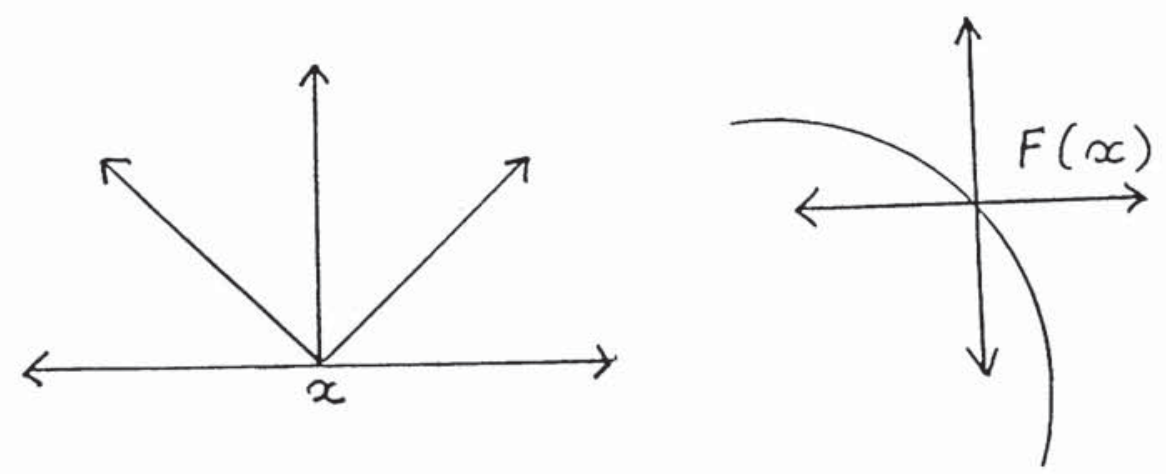

Figure 4

So at least one of them must enter the disk $D(0,|F(x)|)$. For more precision, one may wish to consider eight directions instead of four, since then one of them will point more sharply towards the center of the disk.

If $F^{\prime}(x)=F^{\prime \prime}(x)=0$, but $F^{\prime \prime \prime}(x) \neq 0$, one needs four directions (resp 8), but this time in a sector of angle $2 \pi / 3$, since angles will be multiplied by 3 . If $F^{\prime}(x)=\cdots=F^{(k)}(x)=0, F^{(k+1)}(x) \neq 0$, one needs four directions (resp. 8) in any sector of angle $2 \pi /(k+1)$.

So one can avoid to compute any derivative at all (including $F^{\prime}$ ) : divide a circle around $x$ in $n$ sectors of angle $\frac{2 \pi}{n}$, and take four equidistributed directions in each (or, more simply, take $4 n$ directions on the circle, with angles equal to $\frac{\pi}{2 n}$ ). Then for at least one of them, $|y-x|$ will diminish.

In all cases, we see that we can move $x$ in such a way that $|y-x|$ decreases (much more slowly for a singular point than for a regular point, though). Repeating the procedure, we finally obtain $y=x$, that is a root is found.

\section{Staying in the original disk}

In the first step, when y is computed from $x$ by (6), we know that there is a root of $P$ in the disk of diameter $[x, y]$. However, the way we move 
$x$, in later stages, may very well take us outside this disk, so we may end up on another root than the one originally located. Usually, this has no importance, for people want to find a root, and do not care about a specific disk.

However, it may be useful to keep this extra information in mind. It may be the case, for instance, if we try to locate several roots at the same time (using parallel machines): If we can locate disjoint disks $D_{1}$, $D_{2}, \ldots$ at the beginning and if we are sure that each procedure stays in the corresponding disk, we will end up with distinct roots.

So let's see if we can modify the algorithm so as to stay in the original disk of diameter $[x, y]$.

a) Assume first that $x$ is a regular point. The set of directions $d x$ for which $x$ will remain in the disk is the half plane $H_{1}$, tangent to the disk at $x$.

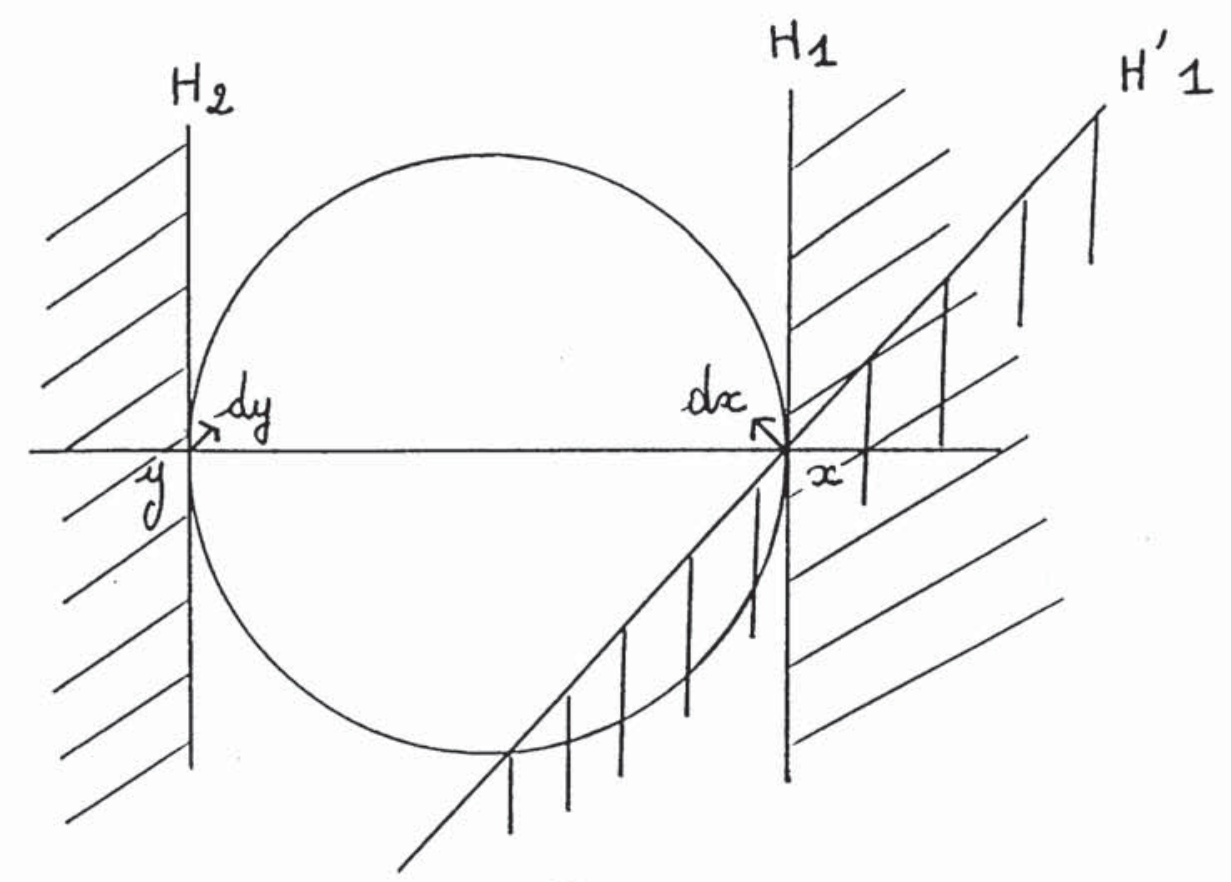

Figure 5

The set of directions $d y$ for which $y$ will remain in the disk is the half plane $\mathrm{H}_{2}$, tangent to the disk at $y$. 
Now, $d y$ is obtained from $d x$ by formula (8), so by a rotation of directions. This means that $H_{2}$ is the image of a half-plane $H_{1}^{\prime}$ passing through $x$. Usually, $H_{1}$ and $H_{1}^{\prime}$ are not equal (both lines intersect at $x$ ) and so, if we take $d x$ in the cone limited by $H_{1}$ and $H_{1}^{\prime}$, both $x$ and $y$ will move into the disk (and thus $|y-x|$ will decrease).

b) The only case where this construction does not work is when $d y=d x$, that is $x$ is a singular point.

Then we will see that, already for a polynomial of degree 3 , one cannot usually stay in a given disk. The example we now detail was already mentioned in $\S 1$.

Let $P=z^{3}+a z^{2}+b z+c$. In symmetrized form, we have the equation

$$
x_{1} x_{2} x_{3}+\frac{a}{3}\left(x_{1} x_{2}+x_{2} x_{3}+x_{3} x_{1}\right)+\frac{b}{3}\left(x_{1}+x_{2}+x_{3}\right)+c=0 .
$$

Taking $x_{1}=x_{2}=x, x_{3}=y$ yields :

$$
x^{2} y+\frac{a}{3}\left(x^{2}+2 x y\right)+\frac{b}{3}(2 x+y)+c=0 .
$$

Let's say we want $y=1$ for $x=0$. For this, we need

$$
b=-3 c \text {. }
$$

Now $P^{\prime 2}(0)-P(0) P^{\prime \prime}(0)=b^{2}-2 a c=9 c^{2}-2 a c$, so 0 will be a singular point if

$$
a=\frac{9}{2} c .
$$

In this case, our polynomial $P$ can be written :

$$
P=z^{3}+\frac{9}{2} c z^{2}-3 c z+c .
$$

If we write $y=x-n F(x)=x+\varphi(x)$, we choose $c$ so that $\varphi^{\prime \prime}(0)=1$. A simple computation gives

$$
\varphi^{\prime \prime}(0)=\frac{2+9 c}{c}, \quad \text { so } \quad c=-1 / 4 .
$$

Taylor expansion at $x=0$ gives :

$$
y-\frac{1}{2}=\frac{1}{2}(x+1)^{2}\left(1-\frac{5 x^{3}}{(x+1)^{2}}+\frac{x^{3} \varepsilon(x)}{(x+1)^{2}}\right), \quad \varepsilon(x) \rightarrow 0, \quad x \rightarrow 0
$$


and so

$$
\left|y-\frac{1}{2}\right|=\frac{1}{2}|x+1|^{2}\left|1-\frac{5 x^{3}}{(x+1)^{2}}+\frac{x^{3} \varepsilon(x)}{(x+1)^{2}}\right| .
$$

Staying in the disk means that $|x-1 / 2|$ and $|y-1 / 2|$ must decrease (or remain the same). But the above formula means that both $|x-1 / 2|$ and $|x+1|$ should decrease, starting at $x=0$, and this is impossible.

So here, in order to decrease $|y-x|$, we have to leave the circle (at least for one of the points). But of course the motion may be arbitrarily small, and this pathological phenomenon will occur only at singular points, that is at $2 n-2$ isolated points. So we can conclude that the algorithm will find a zero in any larger disk : if we start with points $x_{0}, y_{0}$, and fix some $\varepsilon>0$, we can build the algorithm (with small deplacement around singular points) in such a way that it finds the root in the disk of center $\left(x_{0}+y_{0}\right) / 2$, and radius $\frac{1}{2}\left|x_{0}-y_{0}\right|+\varepsilon$.

The algorithm will depend on $\varepsilon$, and the smaller $\varepsilon$ is, the shorter some deplacements will be. Therefore, the algorithm will take longer to converge.

\section{Practical implementation of the algorithm}

Let $P=a_{0}+a_{1} z+\cdots+a_{n} z^{n}$ be a polynomial with complex coefficients, $P^{\prime}$ its derivative. Let $\varepsilon>0$ be a prescribed accuracy.

1. Start at the point $x=-a_{n-1} / a_{n}$, which is the barycenter of the zeros.

- If $P^{\prime}(x)=0$ and $P(x)=0, x$ is a root.

- If $P^{\prime}(x)=0$ and $P(x) \neq 0$, change $x$. Consider for instance one of the $n$ points $x+e^{2 i \pi k / n}, k=1, \ldots, n$.

2. Compute $y=x-n \frac{P(x)}{P^{\prime}(x)}$. Then we know that there is a zero in the disk of diameter $[x, y]$.

3. Let $F=P / P^{\prime}$. Compute $F^{\prime}(x)$.

- If $F^{\prime}(x) \neq 0$, go to step 4 .

- If $F^{\prime}(x)=0$, go to step 7 . 
4. If $F^{\prime}(x) \neq 0$, write $n F^{\prime}(x)$ using polar coordinates :

$$
n F^{\prime}(x)=\rho e^{i \theta}
$$

Let $\theta_{1}=\operatorname{Arg}(y-x)$.

5. Define $h \in \mathbb{C}$ by the properties :

$$
\begin{gathered}
\operatorname{Arg} h=\theta_{1}-\theta \\
|h|=\frac{|y-x|}{\max (\rho, 4)} .
\end{gathered}
$$

6. Replace $x+h$ by $x$, compute the new position of $y$ using step 2. Check that $|y-x|$ has decreased. If not do step 5 again, but with $h$ replaced by $h / 2$ (repeat this step enough times, so that $|y-x|$ actually decreases : this will happen when $h$ is small enough).

7. If $F^{\prime}(x)=0$, compute $F^{\prime \prime}(x)$.

- If $F^{\prime \prime}(x) \neq 0$, write $n F^{\prime \prime}(x)=\rho e^{i \theta}$. Define $h$ by

$$
\begin{aligned}
& \operatorname{Arg} h=\frac{1}{2}\left(\theta_{1}-\theta\right) \\
& |h|=\sqrt{\frac{2|y-x|}{\max (\rho, 4)}}
\end{aligned}
$$

(If $F^{\prime \prime}(x)=0$, go to $F^{\prime \prime \prime} \ldots$ ).

8. Replace $x+h$ by $x$, compute the new position of $y$ using step 2 . Check that $|y-x|$ has decreased. If not, do step 7 again, but with $h$ replaced by $h / 2$; repeat this step enough times.

9. Repeat the procedure, until $|y-x|<\varepsilon$, the prescribed accuracy.

\section{Remarks.}

1. Since the quotient $\frac{|P(x)|}{\left|P^{\prime}\right|}$ can only decrease, and since we started at a point where $P^{\prime} \neq 0$, we are certain that the algorithm will never converge to a zero of $P^{\prime}$ (and, in fact, it will avoid them) unless a zero of $P^{\prime}$ is also a zero of $P$ (that is a multiple zero of $P$ ). 
2. There is some formal similarity with Newton's method: if $x_{n}=x$, then in Newton's method : $x_{n+1}=y$. But he we do not jump from $x$ to $y$, we move them so as to get them closer to each other.

3. To decide to jump from $x$ to $(x+y) / 2$ may be a very bad idea : if $P=z_{1} z_{2}+1, x=1, y=-1$, then $x^{\prime}=(x+y) / 2=0$ and the corresponding $y^{\prime}$ would be $\infty$.

4. In some cases, $y$ may not move when $x$ does. This may happen locally, when $d y=0$, and this means that $(1-n) P^{\prime 2}+n P P^{\prime \prime}=0$ at this point $x$. Or it may happen globally, but only when $P$ is of the form $(x-\lambda)^{2}$. None of these cases prevents the algorithm from working : $x$ gets closer to $y$ and $y$ does not move.

5. When a first $x$ is chosen and $y$ is returned, one more information is obtained, namely that there is a root outside the open disk of diameter $[x, y]$. Indeed, this outside is itself a circular region, which contains both $x$ and $y$ so must contain a root, by Walsh's principle. Therefore, we have the following situation (at any stage of the algorithm): Either there is a root on the boundary circle, or there is a root inside the open disk and a root outside the closed disk.

\section{References}

[1] Beauzamy, B. - Dégot J. Differential Identities. Transactions A.M.S., vol. 347 , July 1995 , no 7, pp. 2607-2619.

[2] Marden, M. : Geometry of polynomials Mathematical Surveys and Monographs. American Mathematical Society, 1966.

[3] Walsh, J. : On the location of the roots of certain types of polynomials. Trans. A.M.S., 24, october 1922, pp. 163-180.

Société de Calcul Mathématique, S. A.

111, Faubourg Saint Honoré

75008 Paris-France

Recibido: 29 de Junio de 1999

Revisado: 24 de Julio de 2000 